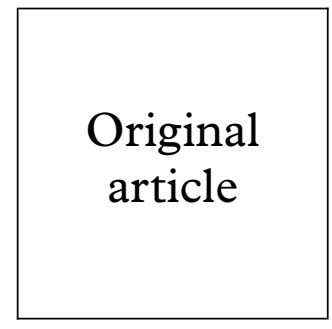

\title{
Auxotypes, serovars, and trends of antimicrobial resistance of Neisseria gonorrhoeae in Kigali, Rwanda (1985-93)
}

Jos Bogaerts, Eddy Van Dyck, Bernadette Mukantabana, Jean Paul Munyabikali, Waldina Martinez Tello

Objective: To investigate the in vitro antimicrobial susceptibility and the auxotype/serovar distribution of Neisseria gonorrhoeae in Kigali, Rwanda, during 1985-93.

Methods: As part of a monitoring programme the in vitro susceptibility of 1604 isolates of $N$ gonorrhoeae was determined by agar dilution. Auxo- and serotyping was performed on 1350 and 1313 isolates respectively.

Results: The prevalence of penicillinase producing $N$ gonorrhoeae (PPNG) remained stable at a rate of 39\% during $1985-91$ and increased to $61 \%$ in $1992-3$. Chromosomal resistance to penicillin was common among non-PPNG and resistance to thiamphenicol and tetracycline was common among both PPNG and non-PPNG. High level, plasmid mediated resistance to tetracycline (TRNG) was observed for the first time at the end of 1989 and increased from $2 \%$ of the isolates in 1990 to $50 \%$ by 1993 . A trend for increasing resistance to norfloxacin and ofloxacin was observed during 1985-90 but disappeared in 1991-93. Five isolates with high level resistance to norfloxacin (MIC $2 \mathrm{mg} / \mathrm{l}$ ) were observed in 1990. Resistance to trimethoprimsulphamethoxazole (TMP-SMZ) emerged at the end of 1990 and was observed among $10 \%$ of the isolates during 1991-3. All strains remained susceptible to ofloxacin, ciprofloxacin, spectinomycin, and ceftriaxone. Overall, $75 \%$ of the isolates were prototrophic or required proline for their growth and $62 \%$ belonged to serovars IA- 6 and IB- 1 . The prevalence of serovar IB- 4 increased strongly during the last 3 years of the study.

Conclusion: Resistance to penicillin, thiamphenicol, and tetracycline was common in $N$ gonorrhoeae during 1985-1993. The rapid spread of TRNG after 1989 and the steep increase of PPNG during 1992-3 were the most striking facts of the study period. The auxotype and serovar distribution was comparable with findings from other African countries.

(Sex Transm Inf 1998;74:205-209)

Keywords: antimicrobial resistance; auxotypes; serovars; Neisseria gonorrhoeae

\section{Introduction}

Neisseria gonorrhoeae infections are an important cause of morbidity among adults and children in Rwanda. ${ }^{1-4}$ Prompt and appropriate antimicrobial treatment is mandatory to eliminate the pathogen, to prevent further complications of the disease, and to limit its transmission. Since resistance patterns of $N$ gonorrhoeae are constantly changing, the monitoring of the in vitro susceptibility of $N$ gonorrhoeae is a prime intervention of public health. In collaboration with the Institute of Tropical Medicine (ITM) in Antwerp, Belgium, a monitoring programme of the in vitro susceptibility of $N$ gonorrhoeae was created in 1983 in the laboratory of the Centre Hospitalier de Kigali (CHK), capital of Rwanda. Trends of antimicrobial resistance obtained previously in the same setting have been published in other papers. ${ }^{5-8}$ In the present paper we analyse the antimicrobial susceptibility patterns and auxotype/serovar distribution of $N$ gonorrhoeae in Kigali during 1985-93.

\section{Materials and methods}

SOURCE OF SPECIMENS

The isolates of $N$ gonorrhoeae, described in this report, were obtained from men, women, and prepubertal girls who presented at the labora- tory of the CHK or the Centre Médico Social de Bilyogo (CMS) during 1985-93 with complaints of urethral/vulvovaginal discharge or with genital ulcers. In addition, $N$ gonorrhoeae was systematically cultured from specimens of conjunctival pus, synovial fluid, pus from pyosalpinx, or Bartholin's gland abscesses which were submitted to the laboratory by other departments of the CHK.

The CHK is a 450 bed general reference hospital serving the city of Kigali (population about 350000 ) and the rest of the prefecture (population about 2 million in 1993). The CMS is a primary health clinic, located at about $4 \mathrm{~km}$ from the CHK in the Nyamirambo quarter, where prostitution is widespread. It serves mainly the lower socioeconomic strata of the population. Patients who presented to the laboratory were interviewed on the use of antibiotics before the visit and the names of the products were noted.

MICROBIOLOGICAL EXAMINATION

Specimens were plated directly onto a modified Thayer-Martin agar where nystatin was replaced by $1 \mathrm{mg} / \mathrm{l}$ amphotericin B. All plates were incubated in a candle jar at $37^{\circ} \mathrm{C}$ during 3 days. A first reading was done after overnight incubation. A presumptive identification was 
done by Gram staining on suspected colonies and by the oxidase test using the p-aminodimethylaniline oxalate reagent. All isolates were tested in the $\mathrm{CHK}$ for the presence of a $\beta$ lactamase using a chromogenic cephalosporin test (Nitrocephin, Unipath, Basingstoke). A total of 4329 isolates of Gram negative, oxidase positive diplococci were cultured and presumptively identified as $N$ gonorrhoeae; 1794 (41\%) of them were penicillinase producing. Isolates were systematically stored in $10 \%$ skimmed milk at $-80^{\circ} \mathrm{C}$ or in liquid nitrogen, except during the months of January, April, July, and August. A total of 940 penicillinase producing and 1203 nonproducing isolates were transported under carbon dioxide ice to the ITM for further testing. Among the surviving isolates, 664 (41\%) were confirmed as PPNG, the remaining 940 as non-PPNG. The survival rate of non-PPNG was significantly better than the rate of PPNG isolates $(78 \%$ or $940 / 1203 v 71 \%$ or $664 / 940$; OR 1.49; $\mathrm{p}<0.001)$. The 1604 surviving isolates were recovered from the cervix ( $\mathrm{n}=$ 737), male urethra $(\mathrm{n}=599)$, vulva of prepubertal girls $(n=121)$, conjunctivae (children and adults) $(\mathrm{n}=110)$, genital ulcers $(\mathrm{n}=$ $35)$, and joint fluid ( $\mathrm{n}=2$ ). Only one $N$ gonorrhoeae isolate per patient was included in the analysis.

\section{ANTIMICROBIAL SUSCEPTIBILITY TESTING AND TYPING}

After identification of $N$ gonorrhoeae, using recommended methods, minimal inhibitory concentrations (MICs) were determined with a multipoint replicator. A final bacterial inoculum of $10^{4}$ colony forming units (cfu) was used. Penicillin, tetracycline, kanamycin, and thiamphenicol were provided by the Laboratory of Standards, Ministry of Health, Brussels (Belgium); norfloxacin by Merck Sharp and Dohme (Brussels); ofloxacin by Hoechst (Brussels); ciprofloxacin by Bayer (Brussels); ceftriaxone, trimethoprim, and sulphamethoxazole (TMP-SMZ) by Roche (Brussels); spectinomycin by Upjohn (Puurs, Belgium), and kanamycin by Bristol (Brussels). The antimicrobial combination of trimethoprim with sulphamethoxazole $(1: 19)$ was tested on diagnostic sensitivity test agar (DST, Oxoid), supplemented with $5 \%$ lysed (freezingthawing) horse blood and 1\% Kellogg's supplement. All other antimicrobials were tested on GC agar base (Difco, Detroit, MI, USA) enriched with $1 \%$ IsoVitaleX (BBL, Cockeysville, MA, USA). Cultures were incubated for 24 hours at $36^{\circ} \mathrm{C}$ in a $5 \%$ carbon dioxide incubator. WHO reference $N$ gonorrhoeae strains A, B, C, D, and E were included at each antimicrobial assay. The following MIC values were interpreted as indicating resistance: $\geqslant 2 \mathrm{mg} / 1$ for penicillin (in non-PPNG), tetracycline, thiamphenicol, norfloxacin, ofloxacin and ciprofloxacin; $\geqslant 128 \mathrm{mg} / \mathrm{l}$ for kanamycin and spectinomycin; $\geqslant 4 / 76 \mathrm{mg} / \mathrm{l}$ for TMP-SMZ. Plasmid mediated tetracycline resistant isolates (TRNG) were presumptively identified when a MIC of $\geqslant 16 \mathrm{mg} / 1$ of tetracycline was observed, and confirmed by the pres- ence of the 25.2 MDa plasmid. ${ }^{9}$ Since MICs of $0.12 \mathrm{mg} / 1$ of norfloxacin represented the highest MIC levels in 1984, before the introduction of norfloxacin in Rwanda, and since MICs of $0.06 \mathrm{mg} / 1$ of ofloxacin and $0.03 \mathrm{mg} / 1$ of ciprofloxacin represented the highest resistance levels of the respective antibiotics in 1986, these MICs were used for the analysis of a trend for increasing resistance over time. Susceptibility to ofloxacin, ciprofloxacin, ceftriaxone, and TMP-SMZ was tested since 1986. Auxotyping and serotyping were performed by standard procedures on isolates obtained since $1986 .^{10}$

\section{STATISTICAL ANALYSIS}

Data were analysed using the EPI-INFO software system (Version 5, Centers for Disease Control, Atlanta, GA, USA). For comparison of proportions the $\chi^{2}$ and, if appropriate, the two tailed Fisher's exact test were used. The odds ratio (OR) was used for measuring associations. Differences between the susceptibility of the predominant auxotypes and serovars were assessed using the Mann-Whitney or Kruskal-Wallis test. The $\chi_{\text {trend }}^{2}$ test was used for the demonstration of a linear trend over time.

\section{Results}

TRENDS OF RESISTANCE

Resistance to individual antibiotics

The annual number of isolates, presumptively identified as $N$ gonorrhoeae, varied between 243 and 906; no trend over time was observed. The rate of penicillinase producing isolates remained fairly stable at $39 \%$ (1533/3898) during 1985-91 and steeply increased to $61 \%$ $(261 / 431)$ in $1992-3 \quad(p<0.001)$. Among strains confirmed as non-PPNG, the rate of chromosomal resistance to penicillin was $19 \%$ (159/842) during 1985-91 and 14\% (11/78) in 1992-3 ( $\mathrm{p}=0.37)$. During the respective periods, decreased susceptibility to penicillin (MIC, $0.5-1 \mathrm{mg} / 1$ of penicillin) was observed among $41 \%(341 / 842)$ and $50 \%(39 / 78)$ of the isolates $(\mathrm{p}=0.06)$.

Overall, $51 \%$ of the non-PPNG and $40 \%$ of the PPNG isolates were resistant to tetracycline (OR 1.57; $\mathrm{p}<0.001)$. High level resistance to tetracycline (MIC $\geqslant 16 \mathrm{mg} / \mathrm{l}$ ) was observed for the first time in December 1989 and increased from $2 \%(2 / 90)$ of the isolates in 1990 to $55 \%$ (43/78) in 1993. During 1990-3, PPNG showed more frequently high level resistance to tetracycline than non-PPNG isolates $(37 \%$ or $81 / 220 v 18 \%$ or $41 / 228$; OR 2.66; $\mathrm{p}<0.001)$.

None of the 30 PPNG isolates and only $2 \%(1 / 52)$ of the non-PPNG showed resistance to thiamphenicol in 1985. During 198693, $53 \%$ of the non-PPNG and $30 \%$ of the PPNG were resistant to thiamphenicol (OR 2.58; $\mathrm{p}<0.001$ ), no trend being observed over time.

A significant trend for increasing resistance to norfloxacin developed among both PPNG and non-PPNG isolates during 1985-90. Only $2 \%(2 / 82)$ of the isolates had MICs of $\geqslant 0.12$ $\mathrm{mg} / \mathrm{l}$ of norfloxacin in 1985 versus $29 \%$ 


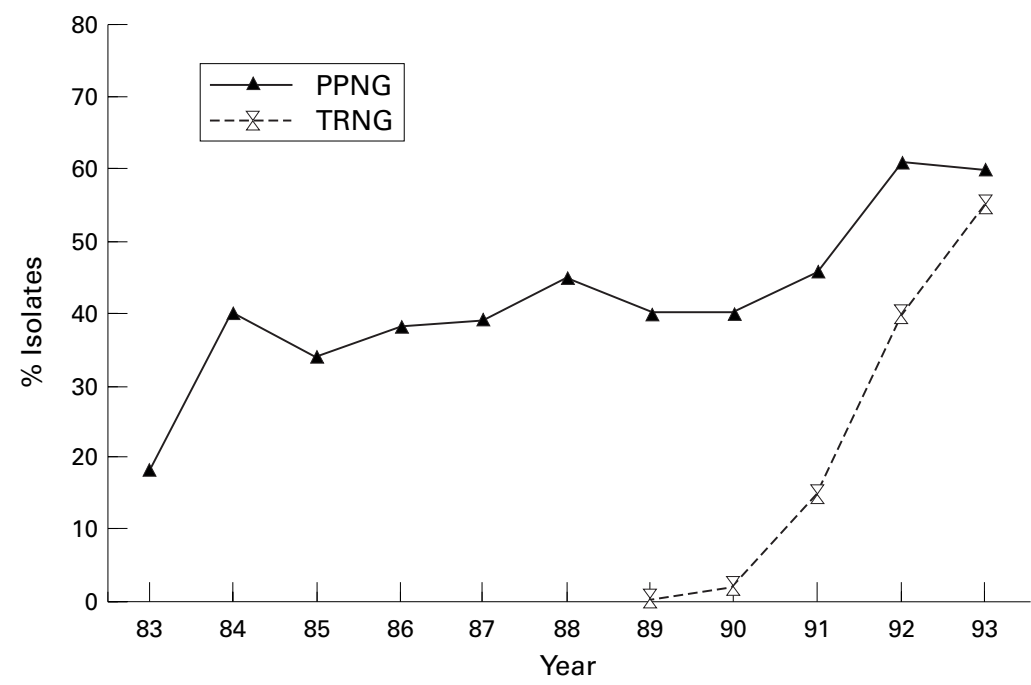

Figure 1 Trends of resistance to penicillin $G$ and tetracycline of Neisseria gonorrhoeae in Kigali (1983-93).
IMPACT OF PREVIOUS ANTIMICROBIAL TREATMENT ON THE IN VITRO SUSCEPTIBILITY OF $N$ GONORRHOEAE

Information on the previous use of antibiotics was available for $14058(80 \%)$ of the 17469 patients who attended the laboratory; of them, $2054(15 \%)$ had taken antibiotics. The products were: penicillin, ampicillin, amoxicillin $(n=925)$, TMP-SMZ $(n=351)$, tetracycline $(n=195)$, spectinomycin $(n=80)$, other agents $(n=260)$, and more than one agent $(n=243)$. The relative frequency of the use of antibiotics remained unchanged over the years. Patients who had taken antibiotics showed more frequently Gram negative, oxidase positive diplococci than patients who had not $(18 \%$ or $369 / 2054 v 16 \%$ or $1917 / 12004$; OR 1.15 ; $\mathrm{p}=0.03)$. The proportion of penicillinase producing isolates was significantly higher in the former group ( $50 \%$ or $183 / 369 v 39 \%$ or $747 /$ 1917; OR 1.5; $\mathrm{p}<0.001)$. Among the surviving isolates, resistance to penicillin (PPNG or chromosomal resistance) was more frequently diagnosed when isolates were obtained from patients with previous treatment than without ( $66 \%$ or $77 / 116 v 51 \%$ or $655 / 1283$; OR 1.89 ; $\mathrm{p}=0.002)$. Similar findings were observed for thiamphenicol $(52 \%$ or $60 / 116 v 41 \%$ or $530 /$ 1283; OR 1.52; $\mathrm{p}=0.04$ ) but no difference was observed for tetracycline $(54 \%$ or $63 / 116 v$ $46 \%$ or $590 / 1283 ; \mathrm{p}=0.10$ ).

\section{AUXOTYPES AND SEROVARS}

Auxotyping was performed on 1350 isolates (573 PPNG, 777 non-PPNG); 49 (4\%) tested isolates did not grow on the complete medium and could not be auxotyped. The prototrophic auxotype (Proto) was observed among $45 \%$ of PPNG versus $32 \%$ of non-PPNG isolates (OR $1.77 ; \mathrm{p}<0.001)$. In contrast, the proline requiring auxotype ( $\mathrm{Pro}^{-}$) was observed among $48 \%$ of the non-PPNG versus $22 \%$ of the PPNG isolates (OR 3.18; p<0.001). The proline requiring/phenylalanine inhibited auxotype was observed among $8 \%$ of PPNG versus $2 \%$ of non-PPNG isolates (OR 5.7; p<0.001) whereas the proline/hypoxanthine/uracil requiring auxotype was observed among $8 \%$ of PPNG and $4 \%$ of non-PPNG (OR 2.31; $\mathrm{p}=0.002)$. All other auxotypes represented less than $5 \%$ of both PPNG and non-PPNG isolates. The relative frequency of the different auxotypes remained fairly stable over time.

Serotyping was performed on 1313 strains (554 PPNG, 759 non-PPNG). Only one isolate was not serotypable. Serovar IA-6 was observed among $38 \%$ of PPNG versus $27 \%$ of non-PPNG (OR 1.68; $\mathrm{p}<0.001)$ whereas serovar IB-1 was observed among $36 \%$ of nonPPNG versus $21 \%$ of PPNG respectively (OR 2.16 ; $\mathrm{p}<0.001)$. Serovars IB-5, IB-4, and IB-7 represented $7 \%, 6 \%$, and $5 \%$ of all the isolates, no difference being observed among PPNG and non-PPNG. The remaining serovars represented less than $5 \%$ of the isolates in each group. Although IA- 6 and IB- 1 were by far the most frequent serovars isolated during the whole study period, their prevalence declined after 1991. In contrast, serovars IB-4 and IB-5, which represented each $2 \%$ of the typed and thiamphenicol. Thirty five per cent (43/ 123) of the TRNG were resistant to thiamphenicol and $72 \%$ to penicillin. 
Table 1 Geometric mean MIC (mg/l) of nine antibiotics for the most frequent A/S classes of $N$ gonorrhoeae in Kigali (1986-93)

\begin{tabular}{|c|c|c|c|c|c|}
\hline & \multicolumn{2}{|l|}{$P P N G$} & \multicolumn{3}{|l|}{ non-PPNG } \\
\hline & $\begin{array}{l}\text { Proto/IA-6 } \\
(n=114)\end{array}$ & $\begin{array}{l}\operatorname{Pro}^{-} / I B-1 \\
(n=45)\end{array}$ & $\begin{array}{l}\text { Proto/IA-6 } \\
(n=63)\end{array}$ & $\begin{array}{l}\text { Pro }^{-} / I B-1 \\
(n=196)\end{array}$ & $\begin{array}{l}\mathrm{Pro}^{-} / \mathrm{IA}-6 \\
(n=74)\end{array}$ \\
\hline Penicillin G & - & - & 1.23 & 1.23 & $0.42^{\star}$ \\
\hline Tetracycline & 0.83 & $2.24^{\star}$ & 0.93 & 2.75 & $1.10^{\star}$ \\
\hline Thiamphenicol & 0.51 & $1.54^{\star}$ & 0.65 & 0.51 & $1.38^{\star}$ \\
\hline Norfloxacin & 0.02 & $0.05^{\star}$ & 0.02 & 0.06 & $0.02^{\star}$ \\
\hline Ofloxacin & 0.01 & $0.02^{\star}$ & 0.01 & 0.03 & $0.02^{\star}$ \\
\hline Ciprofloxacin & 0.003 & $0.008^{\star}$ & 0.003 & 0.01 & $0.003^{\star}$ \\
\hline Ceftriaxone & 0.004 & $0.007^{\star}$ & 0.004 & 0.01 & $0.005^{\star}$ \\
\hline Kanamycin & 19.9 & 18.6 & 17.4 & 20.9 & 16.9 \\
\hline Spectinomycin & 26.3 & $20.4^{\star}$ & 20.8 & 23.4 & 21.9 \\
\hline
\end{tabular}

isolates in 1986 , increased gradually to $24 \%$ and $13 \%$ respectively in 1993 . The auxotype and serovar distribution was similar among men, women, and prepubertal girls.

After exclusion of non-typable isolates, a total of 71 and 82 auxotype/serovar (A/S) classes were identified among the PPNG and non-PPNG isolates respectively. Proto/IA-6 (21\%) and $\mathrm{Pro}^{-} / \mathrm{IB}-1$ (8\%) were the most frequent $\mathrm{A} / \mathrm{S}$ classes among PPNG; among non-PPNG, the most frequent $\mathrm{A} / \mathrm{S}$ classes were $\mathrm{Pro}^{-} / \mathrm{IB}-1$ (26\%), Pro $/ \mathrm{IA}-6$ (10\%) and Proto/ IA-6 $(8 \%)$. The remaining classes represented less than $5 \%$ of the isolates.

ASSOCIATION OF AUXOTYPES AND SEROVARS WITH IN VITRO SUSCEPTIBILITY

During 1986-9, before the appearance of TRNG, $45 \%(134 / 298)$ of serovar IA-6 and $32 \%(95 / 297)$ of serovar IB-1 isolates were PPNG. During 1990-3, the respective rates were $65 \%(79 / 120)$ and $22 \% \quad(20 / 93)$; $(\mathrm{p}<0.001$ and $\mathrm{p}=0.07$ respectively). During 1986-9, serovar IB-4 represented only 3\% $(27 / 906)$ of the isolates versus $14 \%(56 / 407)$ during 1990-3 ( $\mathrm{p}<0.001)$. The prevalence of PPNG among IB-4 isolates increased from $37 \% \quad(10 / 27)$ to $73 \% \quad(41 / 56) \quad(p=0.003)$. Although serovar IB-5 represented 5\% (43/ $906)$ and $13 \%(51 / 407)$ of the isolates during the respective periods $(p<0.001)$, the prevalence of PPNG remained unchanged ( $44 \%$ or $19 / 43 v 39 \%$ or $20 / 51 ; \mathrm{p}=0.78)$. During 1990-93, TRNG were associated with al auxotypes and serovars, except with serovar IB-1.

During both 1986-9 and 1990-3, Pro-/IB-1 isolates were less susceptible to tetracycline, fluoroquinolones, and ceftriaxone than Proto/ IA-6 or Pro-/IA-6 isolates (table 1).

\section{Discussion}

The emergence of TRNG at the end of 1989, and the steep increase of PPNG during 1992-3 were the most striking facts of this observation period. Another interesting fact was the trend for increasing resistance to norfloxacin and ofloxacin during 1985-90 and its subsequent disappearance in 1991-3. In Rwanda, both products were exclusively used for the treatment of gonorrhoea at the CMS clinic of Bilyogo during 1985-9 and were not available elsewhere in the country. ${ }^{8}$ However, the widespread use of nalidixic acid (which is chemically related to the fluoroquinolones) for the treatment of dysentery caused by a multiresistant Shigella dysenteriae type 1 strain during 1981-8, may have contributed to this phenomenon. The emergence of high level resistance to tetracycline was related to the presence of the transferable tet $M$ determinant coding for resistance to tetracycline. TRNG emerged at the end of the 1980s in central Africa and spread rapidly over the continent. ${ }^{12} 13$

Like in other areas of the African continent most PPNG and non-PPNG isolates were prototrophic or proline requiring and a great heterogeneity of auxotypes was observed among both PPNG and non-PPNG. ${ }^{14-20}$

Overall, serovars IA-6 and IB-1 represented $62 \%$ of the strains and their relative frequency remained fairly stable during 1986-91 but declined over the last 2 years of the study. The isolation rate of serovars IB-4 and IB-5, which was less than $5 \%$ until 1991, increased to $24 \%$ and $14 \%$ respectively during 1993 . We postulate that the gradual increase of these serovars is related to the ability to develop antimicrobial resistance through mutation or, more likely, through the acquisition of plasmids coding for antimicrobial resistance. In Rwanda, self treatment is common for all kind of diseases, including sexually transmitted infections, and constitutes a permanent selective antimicrobial pressure favouring the spread of resistant strains. Since culture of clinical specimens, sampling of isolates for transport to the ITM, and the population studied remained unchanged over the years, the increase of serovar IB- 4 and decline of serovar IB- 1 cannot be considered as artefacts. The present data may indicate that the increase of PPNG and TRNG was due to the spread of local strains acquiring new resistant plasmids rather than to the introduction of totally new strains into the community. Further work on the molecular epidemiology of these strains is needed to elucidate the problem.

It must be noted that $8 \%(121 / 1604)$ of the gonococcal strains were cultured from prepubertal girls. Although these children were suspected of being victims of sexual abuse, physical evidence of sexual abuse was not observed. In Rwanda, the belief exists that a man suffering from a purulent urethral discharge can be cured by rubbing his penis on the external genitalia of a prepubertal girl. ${ }^{4}$ In this perspective, auxotyping, as well as genotyping, could have important medicolegal implications but is beyond the scope of a medical laboratory in a developing country. ${ }^{21}$ Although our data probably represent the real susceptibility patterns and the distribution of auxotypes and serovars of $N$ gonorrhoeae in Kigali, interpretation must be cautious since the survival rate of PPNG isolates was significantly lower than the rate of non-PPNG. Since nonsurvivors, especially of PPNG isolates, may belong to a particular auxotype or serovar or exhibit a special type of in vitro susceptibility, these characteristics would not been present in the surviving sample. Loss of isolates is a problem when transporting $N$ gonorrhoeae or other fastidious pathogens to overseas laboratories. 
To avoid these problems, the transfer of technology is essential for the study of the epidemiology of gonorrhoea or other sexually transmitted infections in a developing country, as stated by Mason. ${ }^{17}$

This study was financed in part by the Belgian Administration for Development Cooperation, Brussels, Belgium

Individual contributions from authors not available.

1 Meheus A, De Clercq A, Prat R. Prevalence of gonorrhoea in prostitutes in a central African town. $B r \mathscr{f}$ Vener Dis 1974;50:50-2.

Kestelyn P, Bogaerts J, Meheus A. Gonorrheal keratoconjunctivitis in African adults. Sex Transm Dis 1987;14:191-4

3 Lepage P, Kestelyn P, Bogaerts J. Treatment of gonococcal conjunctivitis with a single intramuscular injection of cefotaxime. F Antimicrob Chemother 1990;26(suppl A):23-7.

4 Bogaerts J, Lepage P, De Clercq A, et al. Shigella and gonococcal vulvovaginitis in prepubertal central african girls. Pediatr Infect Dis f 1992;11:890-2.

5 Piot P, Meheus AZ, Van Dyck E, et al. La sensibilité aux antibiotiques de Neisseria gonorrhoeae au Rwanda. Ann Soc belge Méd trop 1978;58:33-8.

6 Piot P, Van Dyck E, Colaert J, et al. Antibiotic susceptibiliy of Neisseria gonorrhoeae strains from Europe and Africa. of Neisseria gonorrhoeae strains from Europ

7 Bogaerts J, Vandepitte J, Van Dyck E, et al. In vitro antimicrobial sensitivity of Neisseria gonorrhoeae from Rwanda. Genitourin Med 1986;62:217-20.

8 Bogaerts J, Tello WM, Akingeneye J, et al. Effectiveness of norfloxacin and ofloxacin for treatment of gonorrhoea and decrease of in vitro susceptibility to quinolones over time in Rwanda. Genitourin Med 1993;69:196-200

9 Van Dyck E, Rossau R, Duhamel M, et al. Antimicrobial susceptibility of Neisseria gonorrhoeae in Zaire: high level plasmid-mediated tetracycline resistance in central Africa. Genitourin Med 1992;68:111-6.
10 Hendry AT, Stuart IO. Auxanographic grouping and typing of Neisseria gonorrhoeae. Can $\mathcal{F}$ Microbiol 1979;25:512-21.

11 Knapp JS, Tam MR, Nowinski RC, et al. Serological classification of Neisseria gonorrhoeae with use of monoclonal antibodies to gonococcal outer membrane protein I. F Infect Dis 1984;150:44-8.

12 Van Dyck E, Laga M, Manoka AT, et al. Epidemic spread of plasmid-mediated tetracycline resistant Neisseria in Zaire. plasmid-mediated tetracycline resis

13 West B, Changalucha J, Grosskurth $\mathrm{H}$, et al. Antimicrobial susceptibility, auxotype and plasmid content of Neisseria gonorrhoeae in northern Tanzania: emergence of high leve plasmid mediated tetracycline resistance. Genitourin Med 1995;71:9-12.

14 Perine PL, Totten PA, Knapp JS, et al. Diversity of gonococcal plasmids, auxotypes and serogroups in Ghana. Lancet 1983;i:1051-2.

15 Johnson AP, Abeck D, Wall RA, et al. Plasmid content, auxotype and protein-I serovar of gonococci isolated in the Gambia. Epidemiol Infect 1987;99:669-74.

16 Peeters MF, Frost EH, Ossari SA, et al. Antibiotic susceptibility in relation to serogroup and auxotype of Neisseria gonorrhoeae isolates from Gabon. Sex Transm Dis 1987;14 $130-4$

17 Mason PR, Gwanzura L. Characterisation by plasmid profiles, serogroups, and auxotypes of Neisseria gonorrhoeae from Harare, Zimbabwe. Genitourin Med 1988;64: 303-7.

18 Odugbemi TO, Brown ST, Biddle J, et al. Plasmid profile, serogrouping and auxotyping of Neisseria gonorrhoeae isoserogrouping and auxotyping of Neisseria gonorth

19 Lind I, Arborio M, Bentzon MW, et al. The epidemiology of Neisseria gonorrhoeae isolates in Dakar, Sénégal 19821986: antimicrobial resistance, auxotypes and plasmid pro1986: antimicrobial resistance, auxotyp
files. Genitourin Med 1991;67:107-13.

20 Falk ES, Bygdeman SM, Birkeland NK, et al. Genotypes and phenotypes of $\beta$-lactamase producing strains of Neisseria gonorrhoeae from African countries. Genitourin Med 1988;64:226-32.

21 Ross JD. Uses and limitations of gonococcal serotyping. Int f STD AIDS 1996;7:14-18. 